\title{
The endogenous ligand for guanylate cyclase-C activation reliefs intestinal inflammation in the DSS colitis model
}

\author{
Danfeng Lan",2\#, Yunling Wen²\#, Xiangqian Dong2\#, Qin Yang#, Yan Liu², Kunhua Wang4, \\ Hongying $\mathrm{Li}^{2 \otimes}$ and Yinglei Miao ${ }^{2 \square}$
}

YYunnan Key Laboratory of Primate Biomedical Research, Institute of Primate Translational Medicine, Kunming University of Science and Technology, Kunming, Yunnan, 650500, China; 2Department of Gastroenterology, The First Affiliated Hospital of Kunming Medical University, Yunnan Institute of Digestive Disease, Kunming, Yunnan, P.R. China; 'Department of Critical Care Medicine, The First Peoples' Hospital of Kunming, Kunming, Yunnan, P.R. China; 4Department of General Surgery, The First Affiliated Hospital of Kunming Medical University, Kunming, Yunnan, P.R. China

\begin{abstract}
Ulcerative colitis (UC) is a major type of inflammatory bowel disease (IBD) and significantly impacts patient quality of life. Previous research revealed that the guanylate cyclase-C (GC-C) signaling pathway is associated with the severity of UC. We aimed to investigate the effect of the GC-C agonist, guanylin (Gn), on inflammatory injury in mice with colitis. An experimental UC model was established in Balb/c mice. Mesalamine served as a positive control. The $\mathrm{Gn}$ overexpression vector was administered once per day for 1 week. Intestinal permeability of the mice was measured using fluorescein isothiocyanate-dextran after the treatment. Histopathologic grading was estimated to assess the inflammatory injury of the colon. The expression level of crucial mediators of the GC-C signaling pathway (Gn, Ugn and GCC) and tight junction proteins (occludin, claudin-1 and ZO-1) was measured in the colon. Additionally, the level of pro-inflammatory cytokines (IL-8 and TNF-a) in serum was measured. After injecting the UC mice with the Gn overexpression vector, the body weight increased, and the frequency of loose stools and bloody stools was decreased. Intestinal permeability and histopathologic score were significantly reduced $(P<0.05)$. The expression level of GC-C, Gn, Ugn, claudin-1 and ZO-1 was significantly increased $(P<0.05)$. The level of IL- 8 and TNF- $a$ in the serum was significantly decreased $(P<0.01)$. Therefore, the application of $\mathrm{Gn}$ overexpression vector can ameliorate the intestinal inflammatory injury and repair the mucosal barrier in colitis mice, which further suggests the clinical therapeutic potential of GC-C agonists in IBD.
\end{abstract}

Key words: Guanylate cyclase-C; Ulcerative colitis; Lentiviral-mediated; Guanylin; Intestinal mucosal barrier

Received: 12 October, 2019; revised: 20 April, 2020; accepted: 05 May, 2020; available on-line: 07 September, 2020

ఐe-mail: miaoyinglei@yeah.net (YM); licongcong6912@163.com (HL)

"Co-first authors: Danfeng Lan, Yunling Wen, Xiangqian Dong, Qin Yang

Acknowledgements of Financial Support: This work was supported by the National Natural Science Foundation of China (81860105, U1802282, 81670501); Applied Basic Research Projects of Yunnan Province (2019FE001); Yunnan Health Training Project of High Level Talents (H-2018041)

Abbreviations: CGMP, cyclic-guanosine-3', $5^{\prime}$-monophosphate; DSS, dextran sulfate sodium; IBD, inflammatory bowel disease; GC-C, guanylate cyclase-C; Gn, guanylin; STa, heat-stable enterotoxins; TJPs, Intestinal epithelial tight junction proteins; TNBS, trinitrobenzene sulfonic; UC, Ulcerative colitis; Ug, uroguanylin

\section{INTRODUCTION}

Ulcerative colitis (UC) belongs to a group of chronic idiopathic inflammatory disorders that primarily affect the colon and is a major type of inflammatory bowel disease (IBD). IBD significantly impacts patients' quality of life due to its repeated remissions and relapses. While the etiology of these diseases is still not well established, one of the contributing factors may be a poorly regulated immune response against the enteric microbiota in genetically predisposed individuals (Xavier \& Podolsky, 2007). The number of IBD patients has been increasing worldwide (Quaresma et al., 2019). Medical therapies for symptomatic relief of IBD include sulfasalazine, antibiotics, corticosteroids, azathioprine, biologic agents and so on. Although the treatment outcome improved with the use of biologic agents, a certain proportion of patients are resistant to the pharmacological therapy and suffer severe side effects (Ordás et al., 2012; Engel et al., 2010). Therefore, there is a need to identify novel pharmacological targets that act locally at the site of inflammation to maximize the efficacy and minimize the systemic side effects of IBD treatment.

Intestinal epithelial tight junction proteins (TJPs) act in the intestinal epithelium to form a tight mucosal mechanical barrier. Maintenance of the intestinal epithelial cell barrier function is considered to be crucial for the host immune defense. Thus, a dysfunctional barrier and increased gut permeability might be the crucial pathophysiological factors underlying the etiology of IBD. Recent studies suggested that the guanylate cyclase-C (GCC) signaling pathway is involved in the maintenance of a healthy intestinal barrier, anti-inflammatory effects, control of epithelial proliferation and tumorigenesis, and decrease of visceral pain sensation (Camilleri, 2015; Uranga et al., 2018). GC-C is a transmembrane enzyme expressed primarily on the intestinal epithelial cells (IECs) and serves as the receptor for the peptides guanylin $(\mathrm{Gn})$ and uroguanylin (Ugn), as well as the receptor for heatstable enterotoxins (STa) produced by enterotoxigenic Eschericbia coli (Brierley, 2012). The endogenous ligands Gn, Ugn and the exogenous ligands STa of E. coli are structurally related peptides that activate the GC-C receptor, which is selectively expressed on the brush border membranes of enterocytes from the duodenum to the rectum (Forte, 1999). The biologically functional Gn, Ugn peptides are produced in situ in the goblet cells and enterochromaffin-like cells (Li et al., 1995; Perkins et al., 1997). Gn is most abundant in the large intestine 
and distal small intestine, while Ugn expression is higher in stomach and duodenum (Joo et al., 1998). Ligandinduced GC-C activation leads to intracellular elevation of cyclic-guanosine-3',5'-monophosphate (cGMP). This physiological activation of GC-C regulates intestinal fluid and electrolyte homeostasis, preventing dehydration and intestinal obstruction (Basu, 2010).

Previous research showed that the guanylate cyclase- $\mathrm{C}$ signaling pathway was down-regulated in IBD and mice with 2,4,6-trinitrobenzene sulphonic acid (TNBS) colitis (Brenna et al., 2015). Consistent with this study, our study found that the expression level of GC-C, Gn and Ugn in the colonic mucosa of UC patients was significantly decreased compared to normal controls, and this decrease was more significant with increased disease activity, which suggests that the activity of GC-C signaling is reduced in UC patients and negatively correlates with the clinical severity of UC (Lan et al., 2016). In vitro studies revealed that the activity of GC-C signaling pathway can be restored by GC-C agonists. Activation of the GC-C signaling pathway ameliorated the impairment of barrier function and inflammatory injury of Caco- 2 cells induced by interleukin-1 $\beta$ (Wang et al., 2016). Shailubhai and others (Shailubhai et al., 2015) reported that orally administered GC-C agonists plecanatide and dolcanatide, the structural analogs of Ugn, could reduce intestinal inflammatory injury induced by chemical dextran sulfate sodium (DSS) or trinitrobenzene sulfonic (TNBS) in mice (Shailubhai et al., 2015). Chang and others (Chang et al., 2017) showed that orally administered plecanatide reduced the degree of inflammation-driven colonic dysplasia in mice (Chang et al., 2017). In accordance with these findings, Boulete and others (Boulete et al., 2018) indicated that activation of GC-C signaling may be an attractive therapeutic approach to treat functional constipation disorders and inflammatory gastrointestinal conditions (Boulete et al., 2018). Recent studies reported that GC-C agonists plecanatide and linaclotide can be used in the treatment of chronic idiopathic constipation (Lembo et al., 2011; Islam et al., 2018). As we know, diarrhea is the most common manifestation of UC. In this study, we focused on the lentiviral-mediated $\mathrm{Gn}$ expression and performed the experiment in DSS-induced UC mouse model using the $\mathrm{Gn}$ overexpression vector, to determine the potential therapeutic value of $\mathrm{GC}-\mathrm{C}$ agonist in $\mathrm{UC}$. This in vivo study provided powerful experimental evidence for further clinical trials and exploring more efficacious treatments in IBD patients.

\section{MATERIAL AND METHODS}

Animal studies. Sixty male Balb/c mice weighing 18$22 \mathrm{~g}$ were obtained from the Animal Center of Kunming Medical University. All experiments were performed with the approval of the Animal Experimentation Committee of Kunming Medical University and in accordance with the institutional regulations. Mice were divided into 5 groups and the number of mice in each experimental group was 12 . Control group: after normal drinking water for one week, $0.2 \mathrm{~mL} 0.9 \%$ normal saline (NS) was administered by gavage (1 time/day) and $50 \mu \mathrm{L}$ of control vector without Gn $\left(1.776 \times 10^{7}\right.$ copies $\left./ \mathrm{mL}\right)$ was administered by caudal vein injection (1 time/day) for one week. DSS+NS group: after drinking water with 3\% DSS for one week, $0.2 \mathrm{~mL} 0.9 \%$ NS was administered by gavage (1 time/day) for one week. DSS+Mesalamine group: after drinking water with 3\% DSS for one week, $0.2 \mathrm{~mL}$ $30 \mathrm{mg} / \mathrm{kg}$ mesalamine suspension was administered by gavage (1 time/day) for one week. DSS+Gn group: after drinking water with $3 \%$ DSS for one week, $50 \mu \mathrm{L}$ solution of $\mathrm{G} n$ overexpression vector $\left(1.776 \times 10^{7}\right.$ copies/ $\mathrm{mL}$ ) was administered by caudal vein injection (1 time/ day) for one week. DSS+ Mesalamine+Gn group: after drinking water with 3\% DSS for one week, $0.2 \mathrm{~mL} 30$ $\mathrm{mg} / \mathrm{kg}$ mesalamine suspension was administered by gavage (1 time/day) for one week. Concurrently, $50 \mu \mathrm{L}$ of Gn overexpression vector $\left(1.776 \times 10^{7}\right.$ copies $\left./ \mathrm{mL}\right)$ was administered by caudal vein injection (1 time/day) for one week.

Induction of colitis using DSS. The UC mouse model was induced by feeding mice 3\% DSS (Sigma, United States) dissolved in UV-sterilized tap water for seven days. On day seven, all animals were returned to plain water. The successful establishment of the colitis model was indicated by the presence of any of the following symptoms: (1) loose stool, (2) diarrhea, (3) fecal occult blood positive, or (4) rectal bleeding. After DSS administration, the indicators including eating, activity, body weight, stool consistency, and rectal bleeding, were recorded from the first day of the experiment.

Construction of the recombinant lentivirus plasmid for $\mathrm{Gn}$ overexpression. The lentiviral expression vectors for $G$ n protein overexpression and the control vectors without $\mathrm{Gn}$ were purchased from GeneCopoeia (Guangzhou, China). The plasmids were EX-Mm02903Lv109 and EX-NEG-Lv109, and their concentrations were $0.939 \mu \mathrm{g} / \mu \mathrm{L}$ and $0.964 \mu \mathrm{g} / \mu \mathrm{L}$, respectively. First, $293 \mathrm{Ta}$ cells were thawed and subcultured until they reached a density of $90 \%$ and inoculated into a $10 \mathrm{~cm}$ cell culture plate. When the cells grew to a density of $80 \%$, the plasmid was transfected into the $293 \mathrm{Ta}$ cells to package the lentivirus. Afterwards, the lentivirus concentration was measured and the titer was determined. The viral RNA was extracted first, and then DNAse I was used for processing. The qPCR method was used to detect the titer of the plasmid lentivirus after the reverse transcription reaction. The titer was $1.776 \times 10^{7}$ copies/ mL.

Intestinal permeability assay. On the 14th experimental day, at the end of the treatment, the intestinal permeability of mice was measured to assess the barrier function using the fluorescein isothiocyanate-dextran (FITC-D) method. Firstly, mice were anesthetized with ketamine at $8 \mathrm{mg} / 100 \mathrm{~g}$ before the experiment. Then the abdominal wall was incised, the colon was separated $4 \mathrm{~cm}$ away, and both ends of the intestinal canal were clipped with an artery clip covered with a rubber sleeve to prevent the release of the fluorescent compound. 1 mL PBS solution containing 25 mg FITC-D (MW 4000, FD-4, Sigma) was injected into the intestinal lumen. After $2 \mathrm{~h}, 1 \mathrm{~mL}$ blood was collected from the portal vein of the mice, diluted in $50 \mathrm{mM}$ Tris solution and centrifuged at $1000 \mathrm{rpm}$ for $7 \mathrm{~min}$. Then, the supernatant was discarded and the remainders were placed into a 96-well plate specially designed for use in a fluorescence spectrophotometer. The concentration of FD-4 in the samples was detected using a fluorescence spectrophotometer (22331, Millipore, USA); the fluorescence intensity was measured (excitation $480 \mathrm{~nm}$; emission $520 \mathrm{~nm}$ ), and FD-4 concentrations were determined using standard curves generated by serial dilution of FD-4 (Nagy et al., 1989). Permeability was calculated by the linear regression analysis of the sample fluorescence.

Colon histopathologic grading. On the 15 th day of the experiment, the mice were sacrificed by cervical dislocation under anesthesia, the peripheral blood was collected by the eyeball extraction, and the colon tissue was 
collected. In brief, the colon tissue was washed with PBS and soaked in $4 \%$ paraformaldehyde for $48 \mathrm{~h}$, the fixed tissue was soaked in alcohol to dehydrate, and the dehydrated tissue was put in dimethylbenzene. The tissue was soaked and permeated in paraffin, and the paraffinembedded tissue was sliced into about $5-\mu \mathrm{m}$-thick slices. The tissue was baked at $60^{\circ} \mathrm{C}$ in an incubator for 30 min. Then the tissue was hydrated, stained with hematoxylin at $37^{\circ} \mathrm{C}$ for $5 \mathrm{~min}$, and stained with eosin for 3 min. Then it was washed, dehydrated, sealed and taken a picture. The damage of colon tissue was assessed using microscopy by two pathologists in a blinded manner. The inflammation was graded as follows: severity of the epithelial damage (0-2: none, erosion, ulceration), depth of the ulcer (0-3: none, submucosal, muscularis, serosa), edema (0-3: none, slight, moderate, severe), infiltration of the inflammatory cells (0-3: none, slight, moderate, severe), the extent of the infiltration (0-3: none, submucosal, muscularis, serosa).

Immunohistochemistry. Firstly, the tissue section was prepared using a slicer, the thickness of each piece was $5 \mu \mathrm{m}$. The slices were placed in xylene I and II to dewax for $10 \mathrm{~min}$ each, and soaked in anhydrous ethanol I and II for 2 min each. Then, they were rinsed with distilled water and the first antibody was added. The glass slides were put in a wet box and placed in a $4^{\circ} \mathrm{C}$ refrigerator overnight. After rinsing with PBS buffer, the slices were added with the second antibodies respectively, including rabbit polyclonal anti-Gn antibody (1:200, Abcam, USA), goat polyclonal anti-Ugn antibody (1:200, Santa Cruz Biotechnology, USA), mouse monoclonal anti-GC-C antibody (1:250, Sigma, USA), rabbit monoclonal anti-occludin antibody (1:100, Gene Tex, USA), rabbit monoclonal anti-claudin-1 antibody (1:200, Gene Tex, USA), and rabbit monoclonal anti-ZO-1 antibody (1:250, Millipore, USA). Then, the DAB chromogen solution was added to terminate the staining. Finally, the experimental results were observed under an optical microscope, and the brown or brown-yellow particles in the cell membrane or cytoplasm were considered as the protein expression. Four fields of each section were randomly selected under a microscope with a magnification of 200 times, and HPIAS-2000 image analysis software was used for grayscale analysis. The immunohistochemical (IHC) scores were assigned by the percentage of positive cells and the staining intensity in each section. The percentage of positive cells was graded as follows: negative ( 0 points), $1-10 \%$ (1 point), $11-50 \%$ ( 2 points), $51-80 \%$ ( 3 points), $81-100 \%$ (4 points). The staining intensity was graded as follows: negative ( 0 points), weak positive staining (1 point), moderate positive staining (2 points), strong positive staining (3 points). IHC scores were the product of the above two values.

Enzyme-linked immunosorbent assay (ELISA) of the murine serum. The peripheral blood serum of the mice was diluted with the assay diluent. The levels of cytokines (IL-8 and TNF- $\alpha$ ) in serum were measured with commercially available ELISA kits (RayBiotech, Minneapolis, MN, USA) according to the manufacturers' instructions.

Statistics. Experiments were performed at least three times. Data were expressed as the mean $\pm \mathrm{SD}$, and normality was assessed using the Kolmogorov-Smirnov test. Variables with a non-normal distribution were logarithmically transformed before statistical analysis. Independent sample $t$-test was used to compare the means between two groups, while the data among multiple groups were analyzed using one-way analysis of variance (ANOVA) followed by "LSD post hoc" test. Statistical significance was defined as a $P$-value $<0.05$. All statistical analyses were performed using SPSS 17.0 software.

\section{RESULTS}

\section{Changes in the general state and colon morphology of the mice}

The control group mice had frequent activity, active foraging, and formed stools. Each mouse had gained weight by the end of the experiment. The mice in the DSS+NS group had less activity, eating and more loose stools. These mice lost weight by the end of the experiment. The mice of each treatment group were characterized by a better state, eating, and activity; their stools returned to normal gradually following the $10^{\text {th }}$ day of the experiment, and their weight was higher. The body weight change relative to the beginning of the experiment was calculated from day 1 to day 14. DSS caused a reduction in body weight gain, the DSS+NS group mice were relatively smaller at the beginning of the treatment due to DSS administration during the previous week, while mesalamine or Gn treatment significantly alleviated this body weight loss (Fig. 1). There was not significant diarrhea observed in mice receiving treatment with $\mathrm{Gn}$ overexpression vector with 2-5 formed stools per day, without any mucus or blood. The frequency of defecation was higher than in the mice from DSS+Mesalamine group but lower than in DSS+NS group.

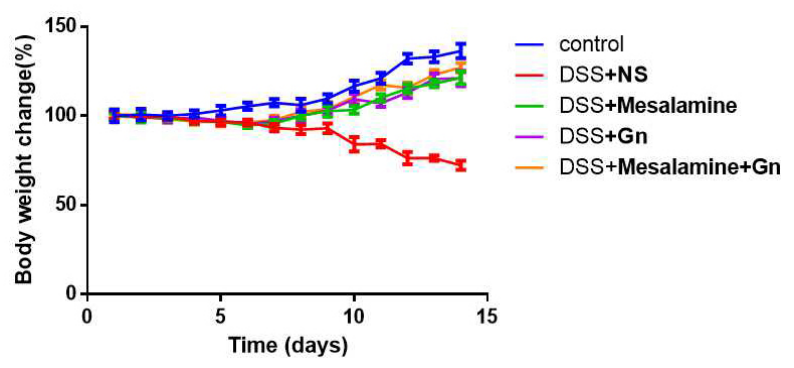

Figure 1. Change of body weight in different groups.

The body weight change relative to the beginning of the experiment was plotted. The percentage was statistically analyzed by chi-square test, the results showed that DSS caused a reduction in body weight gain, the mice from DSS+NS group were relatively smaller at the beginning of treatment due to DSS administration during the previous week, while mesalamine or Gn treatment significantly alleviated this body weight loss.

The appearance, length and general morphology of the mouse colon were assessed by visual observation. It showed that the abdominal cavity of mice in the control group had no adhesion of peritoneum, intestinal tube or mesentery, the intestinal mucosa was smooth and had no ulcers, and there was no thickening in the intestinal wall. The intestines of the DSS+NS group mice were shortened, the bowels were enlarged and had adhesions. There were hyperemia and edema on the surface of the serosa, as well as erosion in the intestinal mucosa without obvious ulcerations; thickening of the intestinal wall and lumen stenosis could be seen. In the mice of each treatment group, the shortening of the colon caused by inflammation was alleviated by the treatment. There were no obvious adhesions nor enlargement of the intestinal tube, and slight hyperemia and edema were observed on the surface of the serosa. Erosions and ulcers could not be observed in the intestinal mucosa (Fig. 2). During 
A

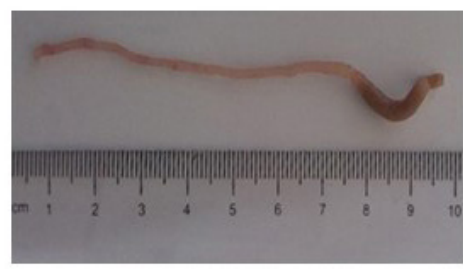

$\mathrm{C}$

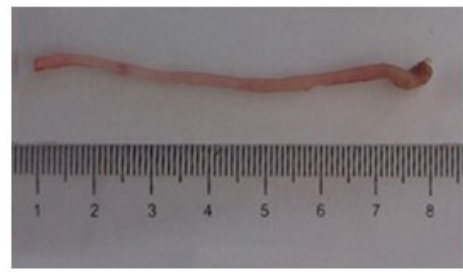

$\mathrm{E}$

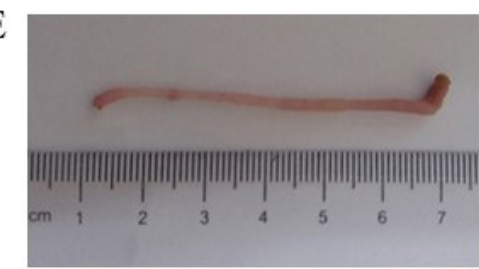

$\mathrm{B}$

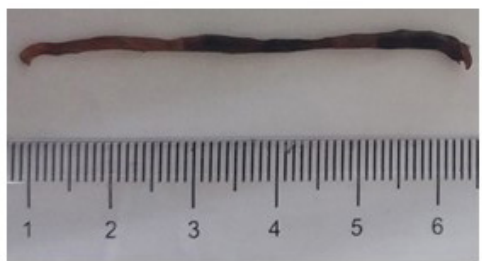

$\mathrm{D}$

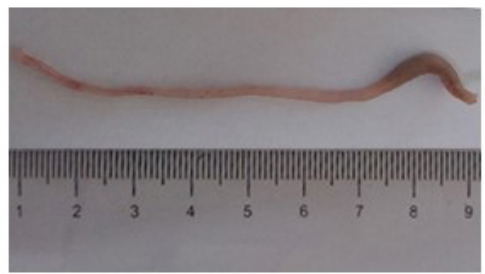

Figure 2. Changes in colon morphology.

A: Control group; B: DSS+NS group; C: DSS+Mesalamine group; D: DSS+Gn group; E: DSS+Mesalamine+Gn group. The appearance, length and general morphology of the mouse colon were assessed by visual observation. It showed that the intestinal mucosa of mice in the control group was smooth and had no ulcers, and there was no thickening in the intestinal wall; The mice in the DSS+NS group had shortened colon, hyperemia and edema at the surface of the serosa, as well as erosion in the intestinal mucosa without obvious ulcerations, thickening of the intestinal wall and lumen stenosis could be seen; The mice of each treatment group had longer colon than the mice of DSS+NS group, they had no obvious adhesions or enlargement of the intestinal tube, and slight hyperemia and edema were observed on the surface of the serosa; erosions and ulcers could not be observed in the intestinal mucosa.

the experiment, 2 mice died in the DSS+NS group and the percentage of dead mice was $17 \% ; 1$ mouse died in the DSS+ Mesalamine group, and 1 mouse died in the DSS + Mesalamine $+\mathrm{Gn}$ group, resulting in the percentage of dead mice of $8 \%$ in both groups.

\section{Changes in Gn, Ugn and GC-C level}

Immunohistochemical analysis showed the expression of Gn, Ugn and GC-C in the intestinal epithelial cells

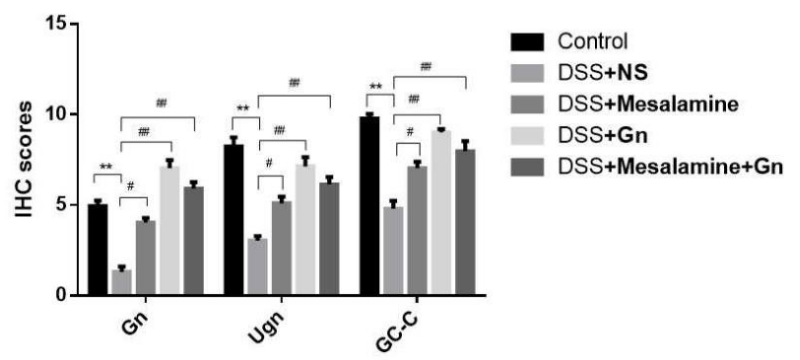

Figure 3. Expression of Gn, Ugn and GC-C in the colon of different groups.

The expression of Gn, Ugn and GC-C in the colon were detected by IHC assay, the IHC scores were graded by the percentage of positive cells and the staining intensity. It was found that the level of Gn, Ugn and GC-C was significantly lower in the DSS+NS group than in the control group but increased significantly after treatments. The increase in Gn, Ugn and GC-C was the most significant in the DSS+Gn group compared to the DSS+NS group. The expression of Gn was higher in DSS+Gn group than in the control. There was no significant difference in the expression of Gn, Ugn and GC-C between the DSS+Gn group and DSS+Mesalamine+Gn group. The number of mice in each group was 12, 10, 11, 12, 11 (from left to right). ${ }^{*} P<0.05,{ }^{* *} P<0.01$ versus the Control group; $\# P<0.05, \# P<0.01$ versus the DSS+NS group. Data are expressed as the mean \pm S.D. All data shown are representative of three independent experiments. of the control group. The level of all the proteins was significantly lower in the DSS+NS group than in the control group $(P<0.01)$ but increased significantly after treatments $(P<0.05)$. The increase in Gn, Ugn and GC-C was the most significant in the DSS + Gn group compared to the DSS+NS group (Fig. 3, Supplementary Figs. 1-3 at https://ojs.ptbioch.edu.pl/index.php/abp). There was no significant difference in the expression of Gn, Ugn and GC-C between the DSS + Gn group and $\mathrm{DSS}+$ Mesalamine $+\mathrm{Gn}$ group $(P=0.15)$.

\section{Changes in IL-8 and TNF- $a$}

The level of IL- 8 and TNF- $\alpha$ was measured in the peripheral blood using ELISA, and the results showed that the level of both proteins was significantly higher in the DSS+NS group than the control group $(P<0.01)$ but decreased significantly after treatments $(P<0.01)$. The decrease in IL-8 and TNF- $\alpha$ level was the most significant in the DSS + Gn group compared to the DSS+NS group (Fig. 4). There was no significant difference in IL- 8 and TNF- $\alpha$ level between the DSS + Gn group and DSS + Mesalamine + Gn group $(P=0.18)$.

\section{Changes in intestinal inflammation and histopathologic grading}

The histopathologic grading of colon damage was scored by two pathologists in a blinded manner. The colonic structure observed under a microscope was clear in the control group, including the mucosa, submucosa, muscularis and serosa. The arrangement of epithelial cells was neat, goblet cells were rich, and the intestinal gland was regular; capillaries and a small number of scattered lymphocytes could be seen in the lamina propria. The mucosa of colon tissue was partially absent, and goblet cells were largely lost in the DSS+NS group. The 
A

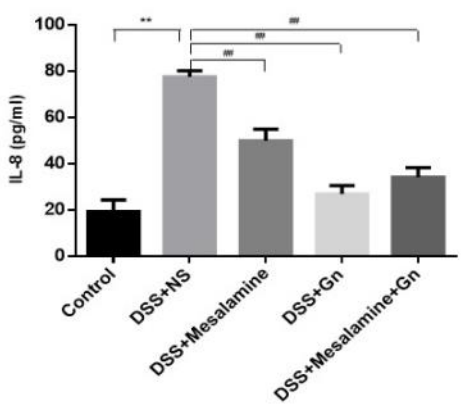

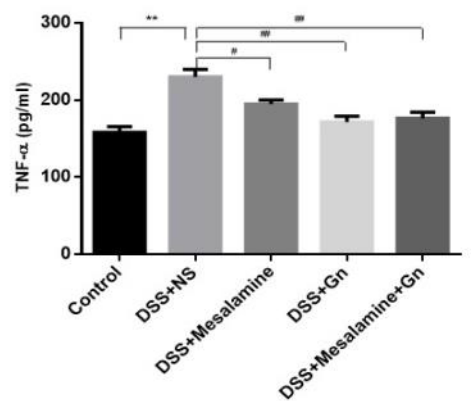

Figure 4. The level of IL-8 and TNF- $a$ in the peripheral blood of different groups.

The level of IL-8 and TNF-a in the peripheral blood serum of mice was detected by ELISA. The results showed that the level of IL-8 and TNF-a was significantly higher in the DSS+NS group than the control group but decreased significantly after treatment. The decrease of IL-8 and TNF-a level was the most significant in the DSS+Gn group compared to the DSS+NS group. There was no significant difference in IL- 8 and TNF-a level between Control and DSS+Gn and between the DSS+Gn and DSS+Mesalamine+Gn group. The number of mice

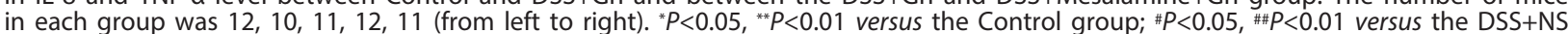
group. Data are expressed as the mean \pm S.D. All data shown are representative of three independent experiments.

A

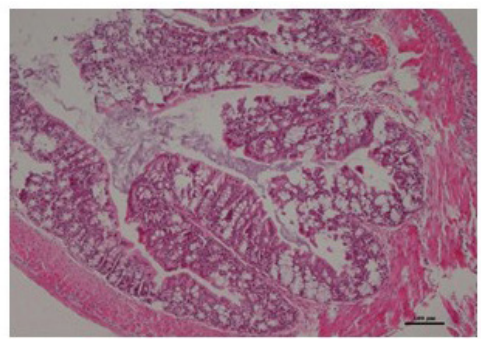

C

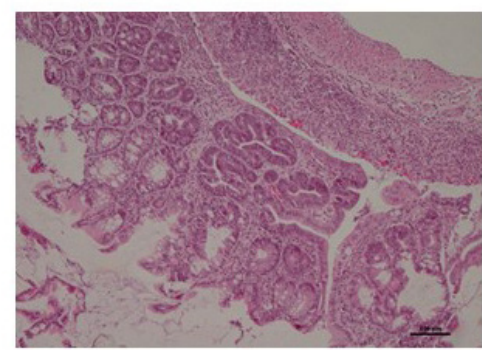

$\mathrm{E}$

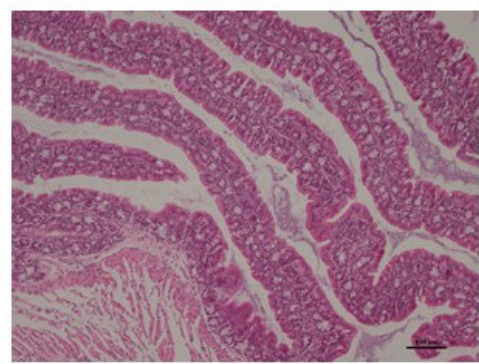

B

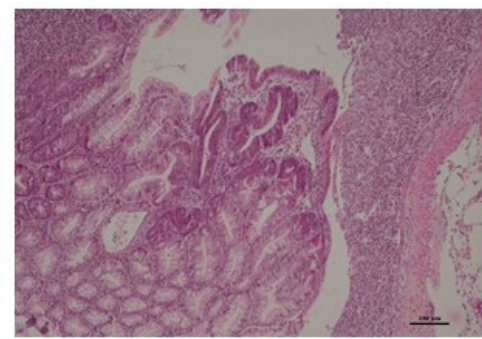

$\mathrm{D}$

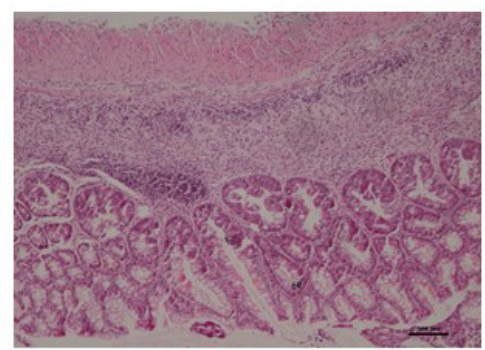

Figure 5. Colonic pathology images of different groups.

The damage of colon tissue was assessed using microscopy by two pathologists in a blinded manner. It showed that the colonic structure was clear in the Control group. The arrangement of epithelial cells was neat, goblet cells were rich, and the intestinal gland was regular, capillaries and a small number of scattered lymphocytes could be seen in the lamina propria; The mucosa of colon tissue was partially absent, and goblet cells were largely lost in the DSS+NS group. After treatment, intestinal inflammation was relieved and epithelial repair was obvious. The irregular regenerative epithelial structure was observed, and inflammatory cells were reduced in the submucosa and muscularis. A: Control group; $\mathbf{B}$ : DSS+NS group; C: DSS+Mesalamine group; $\mathbf{D}$ : DSS+Gn group; E: DSS+Mesalamine+Gn group. The scale bar is $100 \mu \mathrm{m}$.

branched glands and the reparative hyperplasia of the surrounding glands could be seen. Numerous inflammatory cells infiltrated the lamina propria, including neutrophils, lymphocytes, and eosinophils. The histopathologic scores were significantly higher in the DSS+NS group than in the control group $(P<0.01)$. After the treatment, intestinal inflammation was relieved and epithelial repair was obvious. The irregular regenerative epithelial structure was observed, and inflammatory cells were reduced in the submucosa and muscularis (Fig. 5). The decline of the histopathologic scores was the most significant in the DSS + Mesalamine + Gn group compared to the DSS + NS group $(P<0.01)$. There was no significant difference in 


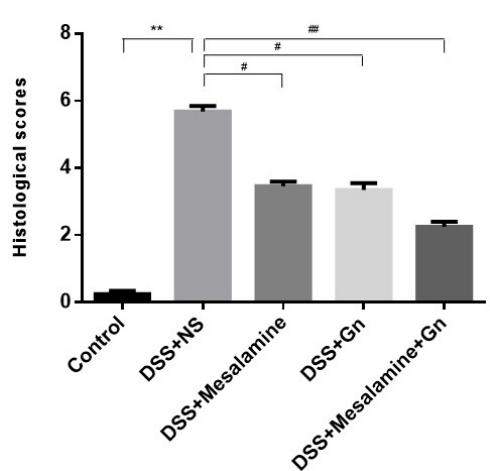

Figure 6. The colonic histopathologic scores of different groups. The histopathologic inflammation scores of colons were graded according to the scoring criteria. It was found that the histopathologic scores were significantly higher in the DSS+NS group than in the Control group but decreased significantly after treatment. The decrease in the histopathologic scores was the most significant in the DSS+Mesalamine+Gn group compared to the DSS+NS group. There was no significant difference in the histopathologic scores between the DSS+Mesalamine group and the DSS+Gn group. The number of mice in each group was $12,10,11,12,11$ (from left to right). ${ }^{*} P<0.05,{ }^{* *} P<0.01$ versus the Control Group; ${ }^{*} P<0.05,{ }^{\# \#} P<0.01$ versus the DSS+NS Group. Data are expressed as the mean \pm S.D. All data shown are representative of three independent experiments.

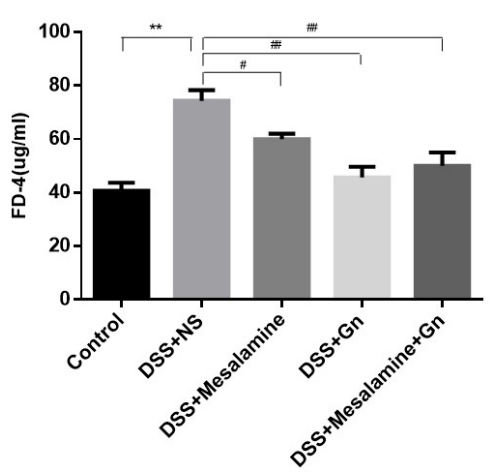

Figure 7. The rate of intestinal passage of FD-4 in the different groups.

FD-4 was injected into the intestinal tract and the passage rate of FD-4 was measured to reflect the intestinal permeability of mice. The results showed that the intestinal permeability was significantly higher in the DSS+NS group than in the Control group but decreased significantly after treatment. The decrease in intestinal permeability was the most significant in the DSS+Gn group compared to the DSS+NS group. There was no significant difference in intestinal permeability between the DSS+Gn group and DSS+Mesalamine+Gn group. The number of mice in each group was $12,10,11,12,11$ (from left to right). ${ }^{*} P<0.05,{ }^{* *} P<0.01$ versus the Control group; ${ }^{\#} P<0.05$, ${ }^{\#} P<0.01$ versus the DSS+NS group. Data are expressed as the mean \pm S.D. All data shown are representative of three independent experiments.

the histopathologic scores between the DSS+Mesalamine group and the DSS $+\mathrm{Gn}$ group $(P=0.12)$ (Fig. 6).

\section{Changes in the intestinal permeability and TJPs}

Intestinal permeability of the mice was evaluated by measuring the rate of intestinal passage of FD-4 macromolecular compound. The results showed that the intestinal permeability was significantly higher in the DSS+NS group than in the control group $(P<0.01)$ but decreased significantly after treatment $(P<0.05)$. The decline in intestinal permeability was the most significant in the DSS + Gn group compared to the DSS $+\mathrm{NS}$ group (Fig. 7). There was no significant difference in intestinal permeability between the DSS $+\mathrm{Gn}$ group and DSS + Mesalamine $+\mathrm{Gn}$ group $(P=0.23)$.

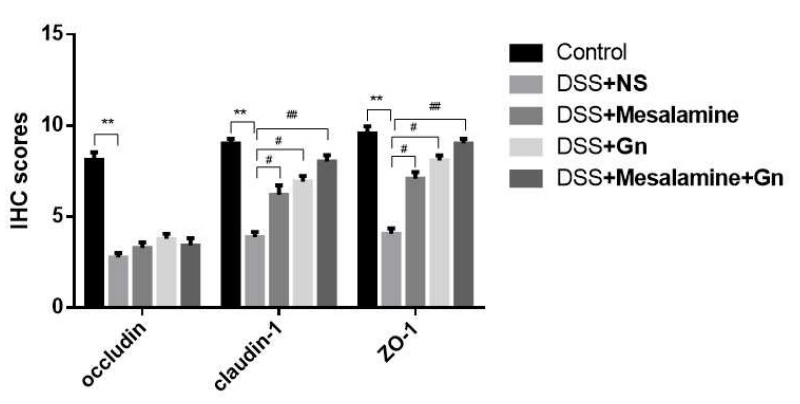

Figure 8. Expression of occludin, claudin-1 and ZO-1 in the colon of different groups.

The expression of occludin, claudin-1 and ZO-1 in the colon was detected by IHC assay, the IHC scores were graded by the percentage of positive cells and the staining intensity. It was found that the level of occludin, claudin-1 and ZO-1 was significantly lower in the DSS+NS group than in the control group. Expression of claudin-1 and ZO-1 increased significantly after treatments, and the increase was the most significant in the DSS+Mesalamine+Gn group compared to the DSS+NS group. There was no significant difference in the expression of claudin-1 and ZO-1 between the DSS+Gn group and DSS+Mesalamine group. The number of mice in each group was $12,10,11,12,11$ (from left to right). ${ }^{*} P<0.05$, ${ }^{* *} P<0.01$ versus the Control group; ${ }^{*} P<0.05, \# P<0.01$ versus the DSS+NS group. Data are expressed as the mean \pm S.D. All data shown are representative of three independent experiments.

The TJPs (occludin, claudin-1 and ZO-1) were expressed in the intestinal epithelial cells of the control group, as determined by immunohistochemistry. The level of all the proteins was significantly lower in the DSS+NS group than in the control group $(P<0.01)$. Expression of claudin-1 and ZO-1 increased significantly after treatments $(P<0.05$ for both proteins), and the increase was the most significant in the DSS +Mesalamine + Gn group compared to the DSS+NS group. There was no significant difference in the level of claudin-1 and ZO-1 between the DSS $+\mathrm{Gn}$ group and DSS+Mesalamine group ( $P>0.05$ for both proteins) (Fig. 8, Supplementary Figs. 4-6 at https://ojs.ptbioch. edu.pl/index.php/abp/).

\section{DISCUSSION}

Treatment of IBD faces great challenges due to the prolonged course and a high recurrence rate. Common therapeutic drugs for IBD include anti-TNF $\alpha$ and antiintegrin agents, glucocorticoids, immunosuppressants, 5-aminosalicylates, enteral nutrition, antibiotics, probiotics, which have limited efficacy and may cause considerable side effects or problems with adherence (Lee et al., 2015). Unresponsive patients may require colectomy, which exposes them to the risk of sequelae, such as pouchitis. With a series of basic studies, many new treatment strategies for IBD emerged, such as stem cell transplantation, fecal microbiota transplantation, and the use of some diets (CDED, CD-TREAT) (Shimizu et al., 2019; Moayyedi et al., 2015; Pigneur et al., 2019). However, fecal microbiota transplantation has very poor efficacy in adults with IBD. Stem cell transplants are not perceived as a great opportunity either. In this study, after treatment of mice with colitis with the Gn overexpression vector, alone or in combination with mesalazine, intestinal permeability was decreased, the general condition of the mice improved and remission of colonic congestion, edema, and adhesion was observed. Histopathologic scores and pro-inflammatory cytokines (IL-8, TNF- $\alpha$ ) 
were reduced. The protein level of crucial mediators of the GC-C signaling pathway (GC-C, Gn, Ugn) and TJPs (claudin-1, ZO-1) was increased. These results suggest that the application of the $\mathrm{Gn}$ overexpression vector alone or in combination with mesalazine recovered the activity of the GC-C signaling pathway and that intestinal inflammation and perturbed barrier function can be mitigated by GC-C agonists. Accordingly, Amarachintha et al. reported that $\mathrm{GC}-\mathrm{C}$ may reduce the invasion of intestinal epithelial cells by bacterial pathogens (Amarachintha et al., 2018).

The endogenous peptides $\mathrm{Gn}$ and Ugn play their role mainly by activating GC-C expressed on the apical surface of intestinal epithelial cells (Forte, 2004). The binding of $\mathrm{Gn} / \mathrm{Ugn}$ to $\mathrm{GC}-\mathrm{C}$ can increase the level of intracellular cGMP, which leads to the secretion of liquid into the gut lumen through a series of signaling reactions. GC-C endogenous ligands function as "fluid sensors", maintaining the balance of water and electrolytes in the intestinal tract and the optimal hydration of the intestinal mucosa (Lucas, 2000). Recent studies showed that orally administered GC-C agonists are rarely absorbed into the systemic circulation and act locally in the gut lumen (Shailubhai et al., 2013; Miner et al., 2013). Previously, we reported that the transcription and expression of GC-C, $\mathrm{Gn}$ and Ugn were downregulated in the colonic mucosa of UC patients and negatively correlated with UC disease activity (Lan et al., 2016). Consistently with our studies, Janecke and others (Janecke et al., 2016) reported that a number of patients with GUCY2C (encoding intestinal receptor $\mathrm{GC}-\mathrm{C}$ ) and SLC9A3 (encoding $\mathrm{Na} / \mathrm{H}$ antiporter 3, a downstream target of GC-C) mutations developed IBD. Primary $\mathrm{Na} / \mathrm{H}$ antiporter 3 and GC-C malfunction is implicated as a predisposition for IBD in a subset of patients (Janecke et al., 2016). In this study, when mice with colitis were treated with a Gn overexpression vector through the caudal vein, the defecation of loose and bloody stools was relieved, and the level of the pro-inflammatory cytokines IL- 8 and TNF- $\alpha$ was significantly reduced in the peripheral blood, indicating that the anti-inflammatory effects of GC-C receptor agonists may be related to their inhibition of the production of pro-inflammatory cytokines.

In addition, our study revealed that GC-C, Gn and Ugn were downregulated in colitis mice, intestinal permeability was decreased, and the expression of claudin-1 and ZO-1 increased significantly after treatment with the Gn overexpression vector, which suggests that the anti-inflammatory effect of the GC-C signaling pathway may be related to the maintenance of the intestinal barrier function. TJPs regulate the barrier function of intestinal epithelial cells (IECs), which control the penetration of pathogens and allergens to the submucosa and beyond (Turner, 2009; Matter \& Balda, 2003). Among many members of TJPs, occludin, claudins and ZO-1 are membrane proteins that connect the adjacent IECs and comprise the epithelial barrier (Mitic \& Anderson, 1998; Ebnet et al., 2004). The TJP occludin behaves differently from claudin and ZO-1. Occludin is important for blocking the gap between adjacent epithelial cells and endothelial cells to maintain the barrier function of tight junctions. The integrity of the intestinal mucosa is maintained by the proliferation, migration, differentiation and apoptosis of IECs (Eastwood, 1992). Previous studies found that GC-C can regulate the proliferation, apoptosis, migration and regeneration of IECs (Lin et al., 2010; Shailubhai, 2002), and aberrant GC-C signaling can lead to the dysfunction of the intestinal epithelial barrier (Mann et al., 2013). The occurrence and severity of co- litis in mice were reduced through drug intervention to protect the intestinal mucosal barrier (Arrieta et al., 2009; Madsen et al., 1999). Similar results were obtained in previous studies, showing that microorganisms in the intestinal lumen infiltrate the intestinal submucosa and below if the intestinal barrier function is impaired, the accumulation of a large number of white blood cells and macrophages disrupts the intestinal homeostasis, leading to the rapid increase of intestine pro-inflammatory factors and promoting the inflammatory reactions in the intestine (Neuman, 2007; Heller et al., 2005). Additionally, it was found that the intestinal permeability of $\mathrm{GC}^{-\mathrm{C}^{-/}}$and $\mathrm{Ugn}^{-/}$mice was higher than that of wild-type mice, and the expression of TJPs in GC-C $-/$ mice was decreased in intestinal mucosa. The GC-C signaling pathway plays an important role in maintaining the balance of proliferation of intestinal epithelial cells and the integrity of intestinal mucosa (Neuman et al., 2007; Steinbrecher et al., 2011).

The limitation of the current study was the failure to detect the level of Gn, Ugn and GC-C in the serum and other tissues, and lack of the group of animals receiving normal drinking water $+\mathrm{Gn}$ overexpression vector solution. The wet and dry weight of feces produced at the end of the study was not recorded. These data will be included in the future study to test the efficacy of GC-C agonists for IBD therapy.

\section{CONCLUSIONS}

Taken together, the present study indicated that the application of a $\mathrm{G} n$ overexpression vector, alone or in combination with mesalazine, can alleviate the inflammatory injury in the colon and restore the barrier function of intestinal mucosa in UC mice. In accordance with our previous findings, these observations further support the notion that the GC-C signaling pathway plays a protective role in intestinal inflammatory injury and epithelial barrier function, and GC-C agonists are a potential treatment approach for UC patients. Nevertheless, excessive activation of GC-C signaling might lead to excessive secretion of water into the gut lumen and cause diarrhea. Future studies will be necessary to demonstrate whether the oral GC-C agonists have therapeutic potential in IBD.

\section{Conflict of interest}

The authors declare no conflict of interest.

\section{REFERENCES}

Amarachintha S, Harmel-Laws E, Steinbrecher KA (2018) Guanylate cyclase $C$ reduces invasion of intestinal epithelial cells by bacterial pathogens. Sci Rep 8: 1521. https://doi.org/10.1038/s41598-01819868-z.

Arrieta M C, Madsen K, Doyle J, Meddings J (2009) Reducing small intestinal permeability attenuates colitis in the IL10 gene-deficient mouse. Gut 58: 41-48. https://doi.org/10.1136/gut.2008.150888.

Basu N, Arshad N, Visweswariah SS (2010) Receptor guanylyl cyclase C (GC-C): regulation and signal transduction. Mol Cell Biochem 334: 67-80. https://doi.org/10.1007/s11010-009-0324-x.

Boulete IM, Thadi A, Beaufrand C, Patwa V, Joshi A, Foss JA, Eddy EP, Eutamene H, Palejwala VA, Theodorou V, Shailubhai K (2018) Oral treatment with plecanatide or dolcanatide attenuates visceral hypersensitivity via activation of guanylate cyclase-C in rat models. World J Gastroenterol 24: 1888-1900. https://doi.org/10.3748/wig. v24.117.1888.

Brenna, $\varnothing$, Bruland T, Furnes MW, van Beelen Granlund A, Drozdov I, Emgård J, Brønstad G, Kidd M, Sandvik AK, Gustafsson BI (2015) The guanylate cyclase-C signaling pathway is down-regulated in inflammatory bowel disease. Scand J Gastroenterol 50: 1241-1252. https://doi.org/10.3109/00365521.2015.1038849. 
Brierley SM (2012) Guanylate cyclase-C receptor activation: unexpected biology. Curr Opin Pharmacol 12: 632-640. https://doi.org/10.1016/j. coph.2012.10.005.

Camilleri M (2015) Guanylate cyclase C agonists: emerging gastrointestinal therapies and actions. Gastroenterology 148: 483-487. https://doi. org/10.1053/j.gastro.2015.01.003.

Chang WL, Masih S, Thadi A, Patwa V, Joshi A, Cooper HS, Palejwala VA, Clapper ML, Shailubhai K (2017) Plecanatide-mediated activation of guanylate cyclase- $C$ suppresses inflammation-induced colorectal carcinogenesis in Apc+/Min-FCCC mice. World J Gastrointest Pharmacol Ther 8: 47-59. https://doi.org/10.4292/wjigt.v8.i1.47.

Eastwood GL (1992) Epithelial renewal in premalignant conditions of the gastrointestinal tract: a review. J Clin Gastroenterol 14: S29-S33. https://doi.org/10.1097/00004836-199206001-00005.

Ebnet K, Suzuki A, Ohno S, Vestweber D (2004) Junctional adhesion molecules (JAMs): more molecules with dual functions? J Cell Sci 117: 19-29. https://doi.org/10.1242/jcs.00930.

Engel MA, Neurath MF (2010) New pathophysiological insights and modern treatment of IBD. J Gastroenterol 45: 571-583. https://doi. org/10.1007/s00535-010-0219-3.

Forte LR (1999) Guanylin regulatory pep- tides: structures, biological activities mediated by cyclic GMP and pathobiology. Regul Pept 81: 25-39. https://doi.org/10.1016/s0167-0115(99)00033-6.

Forte LR (2004) Uroguanylin and guanylin peptides: pharmacology and experimental therapeutics. Pharmacol Ther 104: 137-162. https://doi. org/10.1016/j.pharmthera.2004.08.007.

Heller F, Florian P, Bojarski C, Richter J, Christ M, Hillenbrand B, Mankertz J, Gitter AH, Bürgel N, Fromm M, Zeitz M, Fuss I, Strober W, Schulzke JD (2005) Interleukin-13 is the key effector Th2 cytokine in ulcerative colitis that affects epithelial tight junctions, apoptosis, and cell restitution. Gastroenterology 129: 550-564. https://doi.org/10.1016/j.gastro.2005.05.002.

Islam BN, Sharman SK, Browning DD (2018) Clinical utility of plecanatide in the treatment of chronic idiopathic constipation. Int $J$ Gen Med 11: 323-330. https://doi.org/10.2147/IJGM.S125051.

Janecke, Heinz-Erian P, Müller T (2016) Congenital sodium diarrhea: a form of intractable diarrhea, with a link to inflammatory bowel disease. J Pediatr Gastroenterol 63: 170-176. https://doi.org/10.1097/ MPG.0000000000001139.

Joo NS, London RM, Kim HD, Forte LR, Clarke LL (1998) Regulation of intestinal Cl- and HCO3- secretion by uroguanylin. Am J Physiol 274: G633-G644. https://doi.org/10.1152/ajpgi.1998.274.4.G633.

Lan D, Niu J, Miao J, Dong X, Wang H, Yang G, Wang K, Miao Y (2016) Expression of guanylate cyclase-C, guanylin, and uroguanylin is downregulated proportionally to the ulcerative colitis disease activity index. Sci Rep 6: 25034. https://doi.org/10.1038/srep25034.

Lee D, Albenberg L, Compher C, Baldassano R, Piccoli D, Lewis JD, Wu GD (2015) Diet in the pathogenesis and treatment of inflammatory bowel diseases. Gastroenterology 148: 1087-1106. https://doi. org/10.1053/j.gastro.2015.01.007.

Lembo AJ, Schneier HA, Shiff SJ, Kurtz CB, MacDougall JE, Jia XD, Shao JZ, Lavins BJ, Currie MG, Fitch DA, Jeglinski BI, Eng P, Fox SM, Johnston JM (2011) Two randomized trials of linaclotide for chronic constipation. N Engl J Med 365: 527-536. https://doi. org/10.1056/NEJMoa1010863.

Lin JE, Li P, Snook AE, Schulz S, Dasgupta A, Hyslop TM, Gibbons AV, Marszlowicz G, Pitari GM, Waldman SA (2010) The hormone receptor GUCY2C suppresses intestinal tumor formation by inhibiting AKT signaling. Gastroenterology 138: 241-254. https://doi. org/10.1053/j.gastro.2009.08.064.

Li Z, Taylor-Blake B, Light AR, Goy MF (1995) Guanylin, an endogenous ligand for C-type guanylate cyclase, is produced by goblet cells in the rat intestine. Gastroenterology 109: 1863-1875. https://doi. org/10.1016/0016-5085(95)90753-x.

Lucas KA, Pitari GM, Kazerounian S, et al. (2000) Guanylyl cyclases and signaling by cyclic GMP. Pharmacol Rev 52: 375-414

Madsen KL, Malfair D, Gray D, Doyle JS, Jewell LD, Fedorak RN (1999) Interleukin-10 gene-deficient mice develop a primary intestinal permeability defect in response to enteric microflora. Inflamm Bowel Dis 5: 262-270. https://doi.org/10.1097/00054725199911000-00004.

Mann EA, Harmel-Laws E, Cohen MB, Steinbrecher KA (2013) Guanylate cyclase $\mathrm{C}$ limits systemic dissemination of a murine enteric pathogen. BMC Gastroenterol 13: 135. https://doi.org/10.1186/1471230X-13-135.

Matter K, Balda MS (2003) Signalling to and from tight junctions. Nat Rev Mol Cell Biol 4: 225-236. https://doi.org/10.1038/nrm1055.

Miner PB, Surowitz R, Fogel R, Koltun W (2013) Plecanatide, a novel guanylate cyclase-C (GC-C) receptor agonist, is efficacious and safe in patients with chronic idiopathic constipation (CIC): results from a 951 patient, 12 week, multi-center trial. Gastroenterology 144: S-263. https:// doi.org/10.1016/S0016-5085(13)60585-5

Mitic LL, Anderson JM (1998) Molecular architecture of tight junctions. Annu Rev Physiol 60: 121-142. https://doi.org/10.1146/annurev.physiol.60.1.121.

Moayyedi P, Surette MG, Kim PT, Libertucci J, Wolfe M, Onischi C, Armstrong D, Marshall JK, Kassam Z, Reinisch W, Lee CH (2015) Fecal microbiota transplantation induces remission in patients with active ulcerative colitis in a randomized controlled trial. Gastroenterology 149: 102-109. https:// doi.org/10.1053/j.gastro.2015.04.001.

Nagy JA, Herzberg KT, Masse EM, Zientara GP, Dvorak HF (1989) Exchange of macromolecules between plasma and peritoneal cavity in ascites tumor-bearing, normal, and serotonin-injected mice. Cancer Res 49: 5448-5458. PMID: 2475250

Neuman MG (2007) Immune dysfunction in inflammatory bowel disease. Transl Res 149: 173-186. https://doi.org/10.1016/j. trsl.2006.11.009.

Ordás I, Eckmann L, Talamini M, Baumgart DC, Sandborn WJ (2012) Ulcerative colitis. Lancet 380: 1606-1619. https://doi.org/10.1016/ S0140-6736(12)60150-0.

Perkins A, Goy MF, Li Z (1997) Uroguanylin is expressed by enterochromaffin cells in the rat gastrointestinal tract. Gastroenterology 113: 1007-1014. https://doi.org/10.1016/s0016-5085(97)70198-7.

Pigneur B, Ruemmele FM (2019) Nutritional interventions for the treatment of IBD: current evidence and controversies. Therap Adv Gastroenterol 12: 1756284819890534. https://doi.org/10.1177/ 1756284819890534.

Quaresma AB, Kaplan GG, Kotze PG (2019) The globalization of inflammatory bowel disease: the incidence and prevalence of inflammatory bowel disease in Brazil. Curr Opin Gastroenterol 35: 259-264. https://doi.org/10.1097/MOG.0000000000000534.

Shailubhai K (2002) Therapeutic applications of guanylate cyclase-C receptor agonists. Curr Opin Drug Discov Devel 5: 261-268.

Shailubhai K, Comiskey S, Foss JA, Feng R, Barrow L, Comer GM, Jacob GS (2013) Plecanatide, an oral guanylate cyclase C agonist acting locally in the gastrointestinal tract, is safe and well- tolerated in single doses. Dig Dis Sci 58: 2580-2586. https://doi.org/10.1007/ s10620-013-2684-z.

Shailubhai K, Palejwala, Arjunan K, Saykhedkar S, Nefsky B, Foss JA, Comiskey S, Jacob GS, Plevy SE (2015) Plecanatide and dolcanatide, novel guanylate cyclase-C agonists, ameliorate gastrointestinal inflammation in experimental models of murine colitis. World J Gastrointest Pharmacol Ther 6: 213-22. https://doi.org/10.4292/wjgpt. v6.14.213.

Shimizu H, Suzuki K, Watanabe M, Okamoto R (2019) Stem cellbased therapy for inflammatory bowel disease. Intest Res 17: 311316. https://doi.org/10.5217/ir.2019.00043.

Steinbrecher KA, Harmel-Laws E, Garin-Laflam MP, Elizabeth A Mann, Lucas D Bezerra, Simon P Hogan, Mitchell B Cohen (2011) Murine guanylate cyclase $C$ regulates colonic injury and inflammation. J Immunol 186: 7205-7214. https://doi.org/10.4049/jimmunol.1002469.

Turner JR (2009) Intestinal mucosal barrier function in health and disease. Nat Rev Immunol 9: 799-809. https://doi.org/10.1038/nri2653.

Uranga JA, Castro M, Abalo R (2018) Guanylate cyclase c: a current hot target, from physiology to pathology. Curr Med Chem 25: 18791908. https:// doi.org/10.2174/0929867325666171205150310.

Wang YJ, Lan DF, Niu JK, Miao J, Dong X, Yang G, Zhang F, Wang K, Y. Cao Y, Miao Y (2016) Guanylate cyclase-C signaling pathway regulates intestinal inflammatory injury and epithelial barrier function in Caco-2 cells. Int J Clin Exp Patho 9: 8858-8868.

Xavier RJ, Podolsky DK (2007) Unravelling the pathogenesis of inflammatory bowel disease. Nature 448: 427-434. https://doi. org/10.1038/nature06005. 